\title{
Hybridization for Classification and Identification of Individuals using Multimodal Biometric Systems
}

\author{
Shinde Prashant Pandurang, Sable Amol
}

\begin{abstract}
Biometric recognition systems use certain human characteristics such as voice, facial features, fingerprint, iris or hand geometry to identify an individual or verify their identity. These systems have been developed individually for each of these biometric modalities until they achieve remarkable levels of performance. Biometrics is a measure of biological characteristics for the identification or authentication of an individual based on some of its characteristics. Although biometric recognition techniques promise to be very effective, At present, we can not guarantee an excellent identification rate based on a single biometric signature with unimodal biometric systems. Thus the error rates of unimodal biometric systems are relatively high due to all these practical problems, which makes them impractical for the use of critical safety applications. To resolve these problems, a solution is used in the same system in several biometric modalities, called a multimodal biometric system (MBS). MBSs combine different modalities in a unique recognition system. The multimodal fusion allows improving the results obtained by a single biometric characteristic and making the system more robust to noise and interference and more resistant to possible attacks. Fusion may be carried out at the level of signals acquired by the different sensors, of the parameters obtained for each modality, of the scores provided by unimodal experts or of the decision taken by said experts. In the case of fusion, the features obtained from the various biometric methods must be homogenized before the process of fusion is accomplished. This article describes the evolution of a multi-modal biometric identification system depends on 3 biometrics-face, iris \& fingerprint. Feature extraction is done using the Gabor Wavelet method and classification is accomplished using the Random Forest classifier. This proposed method is applicable in real-life applications to identify biometric for offices, hospitals, and colleges/universities and so on.
\end{abstract}

Keywords: Biometric, Biometric recognition systems, Multimodal biometric systems, fusion, Gabor Wavelet, Random forest.

Revised Manuscript Received on April 25, 2020.

* Correspondence Author

Shinde Prashant Pandurang*, Department of Technology, Savitribai Phule Pune University, Pune, India. Email: prashantshinde09@gmail.com, pshinde@deloitte.com

Sable Amol, Department of Cyber Risk, Deloitte Pune, India. Email asable@deloitte.com

(C) The Authors. Published by Blue Eyes Intelligence Engineering and Sciences Publication (BEIESP). This is an open access article under the CC BY-NC-ND license (http://creativecommons.org/licenses/by-nc-nd/4.0/)

\section{INTRODUCTION}

The identification of individuals corresponds to the search for the identity of the person who appears in a database. It can be used to authorize the use of services, for example, to control access to a highly secure area for which only a limited number of people (registered in a database) have access authorization, as it can be used to recognize criminals. To meet these needs, biometrics seems to be a practical, effective solution whose cost in effort and money is constantly decreasing. Biometrics is developing rapidly. This infatuation leads to the growth of a wide variety of biometric methods: from the classical ones, such as the study of fingerprints [1] or iris [2], to more exotic ones like the recognition of the gait [3], recognition of the shape of the ear [4]. More recently, the increase in identity fraud has created a growing need for biometric technology in several applications needing a high degree of security: access to sensitive sites, airport surveillance [5]. The word biometrics refers in a very broad sense to the measurable study of living things [6]. Biometry is the science that uses mathematics to study biological variations within a given group.

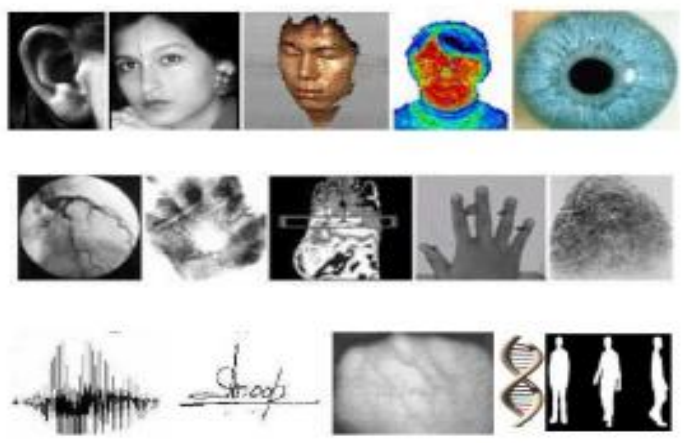

Fig. 1. The different biometric modalities that can serve as a means of identification [7]

Biometric systems can provide three modes of operation, namely, enrolment, identification, \& authentication (or verification). Biometric techniques are currently utilized for security applications. Each has advantages and disadvantages; the choice of a technique is according to the application. Figure 1 shows some biometric techniques. Biometric terms can be classified into three categories:

- Methods that are depends upon the analysis of biological traces (Odor, blood, and DNA);

- Morphological modalities that use part of the human body like iris, fingerprint, etc.

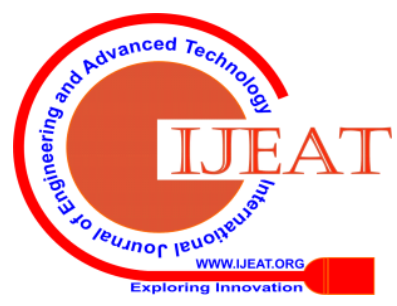


- Behavioral modalities use a personal trait of behavior, such as signature, gait, etc.

Table- I: Advantages and disadvantages of different biometric technologies [8]

\begin{tabular}{|c|c|c|}
\hline Technique & Advantages & Disadvantages \\
\hline Digital print & $\begin{array}{l}\text { Inexpensive; } \\
\text { Average } \\
\text { ergonomics; Ease } \\
\text { of use; Small size } \\
\text { of the reader; The } \\
\text { most tested; Fast } \\
\text { processing }\end{array}$ & $\begin{array}{l}\text { Optimum } \\
\text { quality of } \\
\text { measurement } \\
\text { devices } \\
\text { (reliability); } \\
\text { Average } \\
\text { acceptability; } \\
\text { Possibility of } \\
\text { attacks. }\end{array}$ \\
\hline Geometry of the hand & $\begin{array}{l}\text { Very ergonomic; } \\
\text { Good acceptability }\end{array}$ & $\begin{array}{l}\text { Bulky system; } \\
\text { expensive; } \\
\text { Possible } \\
\text { disturbance by } \\
\text { injuries. }\end{array}$ \\
\hline Face & $\begin{array}{l}\text { Inexpensive; } \\
\text { Compact; Good } \\
\text { acceptability }\end{array}$ & $\begin{array}{l}\text { Identical twins; } \\
\text { Psychology; } \\
\text { Vulnerability to } \\
\text { attacks }\end{array}$ \\
\hline Retina & $\begin{array}{l}\text { Reliability; } \\
\text { Durability }\end{array}$ & $\begin{array}{l}\text { Expensive; Low } \\
\text { acceptability }\end{array}$ \\
\hline Iris & $\begin{array}{l}\text { Reliability. } \\
\text { Durability }\end{array}$ & $\begin{array}{l}\text { expensive; Low } \\
\text { acceptability; } \\
\text { Acquisition } \\
\text { constraints. }\end{array}$ \\
\hline Voice & Ease. & $\begin{array}{l}\text { Vulnerability to } \\
\text { attacks }\end{array}$ \\
\hline Signature & Ergonomics & $\begin{array}{l}\text { Psychology; } \\
\text { Depends on the } \\
\text { reliability of the } \\
\text { signature }\end{array}$ \\
\hline Keyboarding & Ergonomics & $\begin{array}{l}\text { Keyboarding } \\
\text { Physical and } \\
\text { psychic state }\end{array}$ \\
\hline
\end{tabular}

There are many possibilities for improvement in biometrics [9]. Biometric systems that operate using any feature alone (Unimodal biometric systems) have some limitations [10].

The use of multiple biometric systems is multimodality [11]. Several systems together are designed to reduce previous limitations. Also, the use of different systems is primarily aimed at increasing the efficiency of recognition. The goal is to increase the acceptance of the program by increasing the amount of discriminating information for each person. Multimodal systems combine several biometric systems and therefore require the acquisition and processing of several data. The acquisition and the processing can be done successively, one speaks then of architecture in series, or simultaneously, one speaks then of architecture in parallel.

\section{LITERATURE SURVEY}

In the field of people recognizing iris, John Daugman is the pioneer. In 1994, he suggested a complete iris imaging method [12]. Based on calculating an integrodifferential operator, Daugman suggested a system for detecting iris, pupil and eyelids. He then created the "rubber-sheet" approach to normalize the iris disk pseudo-polar. The extraction process of the features is based on the 2D Gabor phase coding. Distance hamming is used to check.

In 2018, Chen et al. [13] The alternative approach suggested and different from Daugman's. Differences include iris acquisition, segmentation, normalization and identification in all processing phases. Chen et al. suggested a system based on the elliptical and circular transform of Hough in segmentation. This device encodes the structure of the iris using the degradation Laplace pyramid. The growing association between 2 iris codes is used to assess the similarity.

In 2016, Shaik [14] Proposed the use of the intensity values for the collection of circular contours clustered on a center of the pupil located using content-sensing techniques to extract a set of one-dimensional signals from the Irish image. The set of one-dimensional signals is then encoded with the transformation "zero crossing" at different levels of resolution. We use the mean of the difference at each degree of resolution to measure the overall dissimilarity between two iris codes.

In 2013, Thepade [15] Wavelet Transform used by a hybrid wavelet transforms. 4 transformation rates are determined and the signature is made up of the lowest diagonal description coefficients.

In 2012, Galbally [16] The shortcomings of iris recognition systems were explored and a correlation approach for the calculation of local quality was proposed when fewer constraints are placed on images, including a loss of quality of an image.

In 2009, Arivazhagan et al. utilize a method known as multiresolution [17]. By calculating the mean grayscale value as a radius function, the ICA Multiresolution Algorithm provides a one-dimensional iris signature that is used as input. Prabhakar et al. [18] propose a method depends upon filter banks to perform the matching of this kind of image. They describe a new texture descriptor called "fingerprint code". The texture information (averages and variances) is extracted by performing a sectorization of the image around a reference point (core point), and pairing is based on a calculation of Euclidean distance between two codes. The disadvantage of this approach is that the core point must be located exactly. This is not possible for poor quality images. Also, the performance is lower compared to matches based on minutiae. However, a decision that combines texture settings and minutiae would have a better performance.

M. T. Leung et al. introduced in [19] neural network for the detection of minutiae. Another more direct approach was proposed by Maio and Maltoni in [20]. This approach tracks the ridges by locating the local maxima by tracking lines along the directional flow of the ridges. Only with this approach, the local maxima may not be well defined in the case of a poor quality image.

The proposed research work depends upon the work of Walia et al. (2019) [21]. They presented a research paper on multimodal biometric identification depending upon score level fusion of iris, fingerprint \& finger-vein images. They used Back-tracking Search Optimization Algorithm for the classification of extracted features.

\section{PROPOSED METHODOLOGY}

\section{A. Problem Statement}

Biometrics is a measure of biological characteristics for the identification or authentication of an individual based on some of its characteristics.

Published By:

Blue Eyes Intelligence Engineering

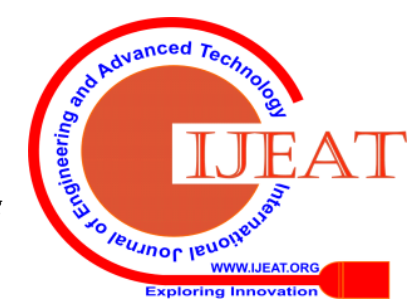


This technique is used more and more today to establish the recognition of the person in a large number of diverse applications. Although biometric recognition techniques promise to be very effective, we cannot presently guarantee an excellent recognition rate by unimodal biometric systems, depending upon a single biometric signature. Also, these systems are generally affected by issues like noise introduced by sensor; non-universality; lack of individuality \& unchanging representation \& sensitivity to attack. Thus, because of all these practical issues, error rates related by unimodal biometric systems are relatively high, which creates them unacceptable for deployment of important security applications. To overcome these problems, a solution is used in the same system in many biometric modalities, named MBS. This research work presented a multimodal identification system combining information from the face, iris, and fingerprint. Since when they are fused they present a better performance and give greater security in practical applications, thus being more reliable and robust in real applications. The results obtained to improve the identification of individuals.

\section{B. Pre-Processing Block}

The pre-processing step initially transforms the RGB image into a grayscale image. Then morphological operations are performed on the grayscale image.

\section{Morphological Operations}

The development of mathematical morphology was inspired by image processing problems, which is its main field of application. In particular, it provides tools for filtering, segmentation, quantification and image modeling. In what follows, we present the basic elements of mathematical morphology. We will study the mathematical morphology applied to binary images (two levels of gray) because this corresponds to our use of this method. However, the approach can also be applied to images in several gray levels.

\section{a) Tools of Mathematical Morphology}

The mathematical morphology is known by its set elements; where $X$ is the set to be analyzed and $S$ a structuring element that one chooses according to the needs of the analysis. These relationships are indeed based on basic morphological operators that are erosion and dilation. On a binary image, $X$ can be the set of white pixels or any subset of the latter, and $S$ any subset of all the sites [22].

$X t=\{x t, x \in X\}$, translated from $X$

$S=\{-S, s \in S\}$, the symmetric of $S$ concerning the origin.

Addition of Minkowski: The addition of Minkowski of the set $X$ by the set $Y$ is defined by [22]:

$$
X \oplus Y=\{x+y, x \in X, y \in Y\}
$$

Subtraction of Minkowski: The Minkowski subtraction of the set $X$ by the set $\mathrm{Y}$ is defined by [22]:

$$
X \ominus Y \cap X Y
$$

Erosion: The mathematical erosion by a structuring element is a subtraction of Minkowski. The structuring element (erosion kernel) determines how erosion tinges borders in an image [22]. The MATLAB command is given by:

$$
g=(f, s e)
$$

Dilation: The mathematical dilation by a structuring element is an addition of Minkowski by $S$ such that:

$$
X \oplus S=\{x, S x \cap X \neq \emptyset\}
$$

The MATLAB command is given by:

$$
g=\text { imdilate }(f, s e)
$$

The Entecavir Test Solution was ready by moving $10 \mathrm{mg}$ of the sample to a volumetric flask of $10 \mathrm{ml}$ and then adding $1 \mathrm{ml}$ of DMSO, Catalyst Solution, and Derivatizing Reagent. Marking with Acetonitrile then extracts this solution.

\section{Feature Extraction using Gabor Wavelet}

Gabor wavelet features are measured for proposed work. The directional decompositions for image analysis were probably initiated by Gaussian windowing in the 2D Fourier domain and continued 4D filters as well as the directional Gabor wavelets proposed by J. Daugman [23]. The use of Gabor's wavelets for the image is often linked to the consideration of the human visual system.

Gabor wavelets are constructed by isotropic Gaussian windowing of a complex plane wave of frequency $F$ in direction $\theta$ [24]:

$$
\psi \theta(x)=e-\|x\| 2 / 2 / 2 \pi e-j(x T \omega 0)(6)
$$

Where, $\omega 0=F[\cos (\theta) ; \sin (\theta)] T$

The decomposition depends on the number of orientations $K$ that one fixes and that one can distribute evenly in $[0, \pi]$.

$$
\theta \in \Theta=\{k \pi / K ; 0 \leq k \leq K\}
$$

We can then decompose any real 2D signal (x) by scalar product with the following atoms:

$$
\{\psi j, u \theta(x)=2-j \psi \theta(2-j(x-u))\} \theta \in \Theta, j \in \mathbb{Z}, u \in \mathbb{R} 2
$$

\section{E. Classification by using Random Forest Classifier}

In particular, methods that utilize randomness to create diversity in tree sets have multiplied. Breiman presented a formal framework for this group of methods that he named RF (Random Forest). An RF is a classifier comprising a set of elementary classifiers of the decision tree type, noted:

$$
\{h(x, \Theta k), k=1, \ldots L\}
$$

Let $\left(h^{\gamma}(\Theta 1), \ldots, h^{\gamma}(\Theta \mathrm{q})\right)$ a collection of tree predictors, with $\Theta 1, \ldots, \Theta q$ random variables independent of $\mathcal{L} n$. The predictor of random forests

$h R F$ is obtained is aggregating this collection of random trees as follows:

Published By:

Blue Eyes Intelligence Engineering

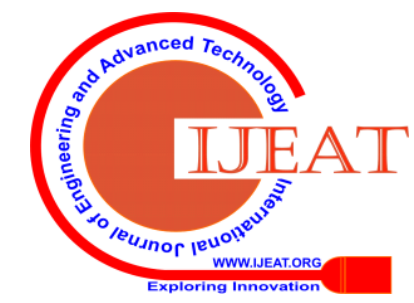


$h R F(x)=1 / q\left(\sum l=1 q h(x, \Theta l)\right)$ Average of individual tree predictions in regression.

$h R F(x)=\arg \max 1 \leq k \leq K \sum l=1 q 1 h^{\prime}(x, \Theta l)=\mathrm{k}$ Majority vote among individual predictions trees in classification.

The term random forest comes from the fact that individual predictors are, here, explicitly predictors per tree, and that each tree depends on an additional random variable (that is, in addition to $\mathcal{L} n$ ).

\section{F. Pseudo Code}

Algorithm 1: Pre-Processing for Fingerprint Recognition Input: Biometric sample, Fingerprint Image " $F P i=f p 1$, fp2. . fpn"

Output: Pre-processed image“ $F P i^{\prime}$ ”.

1. Begin

2. For each biometric sample of fingerprint image

3. Apply Morphological operation using Equations (3) and (5)

4. End for

5. End

Algorithm 2: Feature Extraction for Fingerprint Recognition

Input: Pre-processed image " $F P^{\prime} i$ "

Output: Extracted features " $F P i$ " " in the form of amplitude and energy.

1. Begin

2. For each pre-processed image " $F P i^{\prime}$ " with number of orientation' $k$ '

3. Define the value of $\theta$ with $\theta 1, \ldots, \theta k$ using Gabor filter

4. End for

5. End

Algorithm 3: Pre-Processing for Iris Recognition Input: Biometric sample, Iris Image " $I i=I 1, I 2, \ldots, I n$ "

Output: Pre-processed image " $I i^{\prime \prime}$ ".

1. Begin

2. For each biometric sample of iris image

3. Apply segmentation for pre-processing

4. End for

5. End

Algorithm 4: Feature Extraction for Iris Recognition

Input: Pre-processed image " $I i$ " .

Output: Extracted features " $I i$ " " in the form of amplitude and energy.

1. Begin

2. For each pre-processed image " $I i$," with several orientation ' $k$ '

3. Define the value of $\theta, \theta 1, \ldots, \theta k$ using Gabor filter

4. End for

5. End

Algorithm 5: Feature Extraction for Face Recognition

Input: Biometric sample face image " $F i=f 1, f 2, . ., f n$ "

Output: Extracted features " $F i^{\prime}$ " in the form of

amplitude and energy.

1. Begin

2. For each pre-processed image " $F i$ " with several orientation ' $k$ '

3. Define the value of $\theta, \theta 1, \ldots, \theta k$ using Gabor filter

4. End for

5. End

\section{Algorithm 6: Classification}

Input: Biometric features extracted, $F P i^{\prime}$, " $I i^{\prime \prime}$, " $F i^{\prime \prime}$

Output: Result in terms of accuracy, precision, and sensitivity.

1. Begin

2. For $i=1: n, n=$ number of training samples

3. Create classifier model using Bagger tree

4. End for

5. For $i=1: n, n=$ number of testing samples

6. Predict classifier model

7. End For

8. Calculate accuracy, precision, and sensitivity

9. End

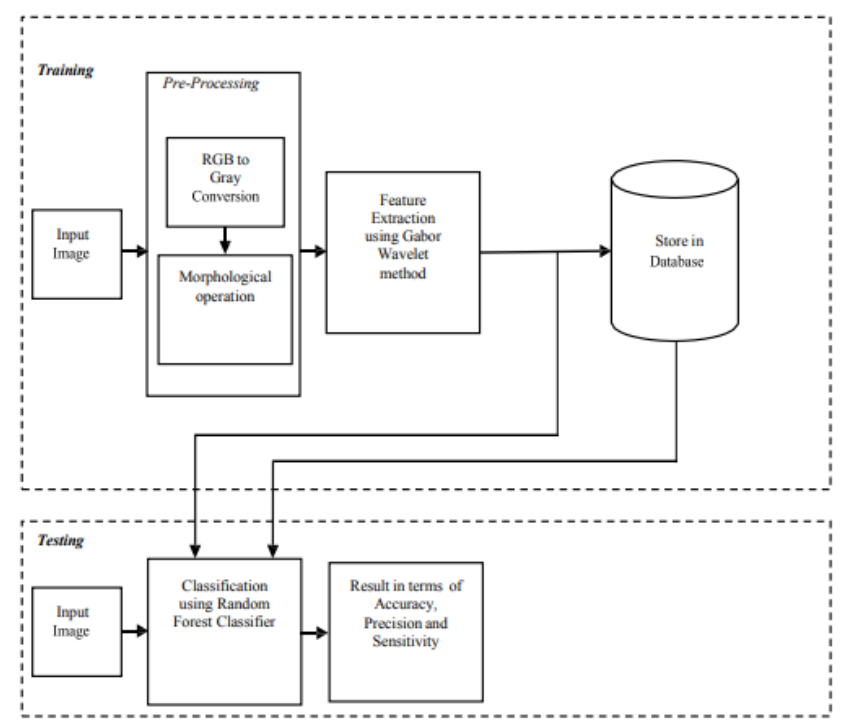

Fig. 2. Block diagram for proposed multimodel biometric identification system

There are three input patterns for proposed multimodal biometric identification i.e. face, iris, and fingerprint images. Figure 2 shows the proposed system modal containing training and testing modules.

\section{EXPERIMENTAL RESULTS}

Simulation is carried out by with MATLAB software image processing (IP) toolbox.

\section{A. Evaluation Parameters}

Accuracy $=((T P+T N) /(T P+T N+F P+F N)) \times 100$
Precision $=(T P /(T P+F P)) \times 100$
Sensitivity $=(T P /(T P+F N)) \times 100$

Where,

$$
\begin{aligned}
& \text { TP=True Positive } \\
& \text { TN=True Negative } \\
& \text { FP=False Positive } \\
& \text { FN=False Negative }
\end{aligned}
$$


B. Simulation Results and Analysis

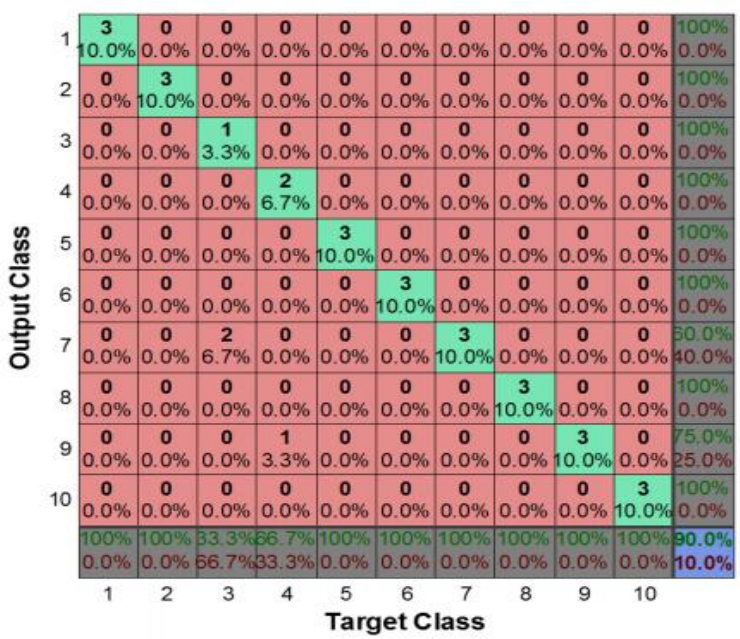

Fig. 3. Confusion matrix plot for face recognition

Table- II: Result for face recognition for different classes

\begin{tabular}{|l|l|l|l|l|l|l|l|l|l|l|}
\hline \multirow{2}{*}{ Parameter } & \multicolumn{10}{|c|}{ Class } \\
\cline { 2 - 12 } & $\mathbf{1}$ & $\mathbf{2}$ & $\mathbf{3}$ & $\mathbf{4}$ & $\mathbf{5}$ & $\mathbf{6}$ & $\mathbf{7}$ & $\mathbf{8}$ & $\mathbf{9}$ & $\mathbf{1 0}$ \\
\hline TP & 3 & 3 & 1 & 2 & 3 & 3 & 3 & 3 & 3 & 3 \\
\hline FP & 0 & 0 & 2 & 1 & 0 & 0 & 0 & 0 & 0 & 0 \\
\hline FN & 0 & 0 & 0 & 0 & 0 & 0 & 2 & 0 & 1 & 0 \\
\hline TN & 27 & 27 & 27 & 27 & 27 & 27 & 25 & 27 & 26 & 27 \\
\hline
\end{tabular}

Calculation of confusion matrix for different classes is given as follows:

For Class 1:

$$
\begin{aligned}
\mathrm{TP}=3, \mathrm{TN} & =27, \mathrm{FP}=0, \mathrm{FN}=0 \\
\text { Accuracy } & =T P+T N / T P+T N+F P+F N \\
& =3+27 / 3+27+0+0=100 \%
\end{aligned}
$$

Precision $=T P / T P+F P=3 / 3+0=100 \%$

Sensitivity $=T P / T P+F N=3 / 3+0=100 \%$

Similarly, to all other remaining classes this calculation will have performed.

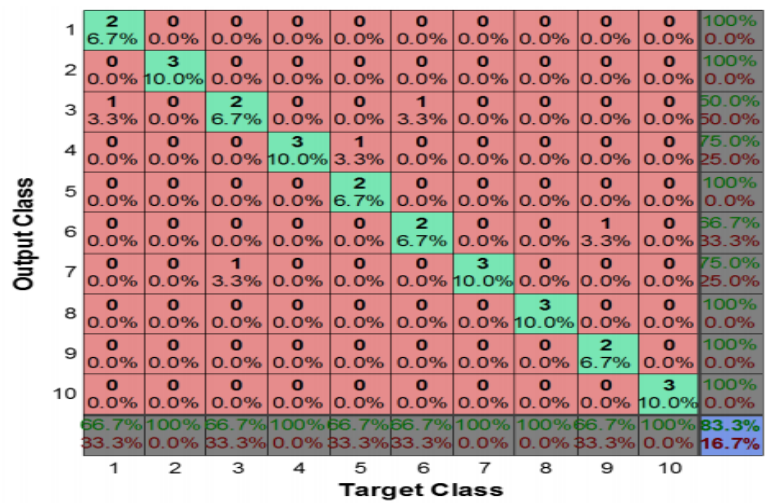

Fig. 4.Confusion matrix plot for iris recognition approach

Table- III: Result for Iris recognition approach for different classes

\begin{tabular}{|l|l|l|l|l|l|l|l|l|l|l|}
\hline \multirow{2}{*}{ Parameter } & \multicolumn{10}{|c|}{ Class } \\
\cline { 2 - 12 } & $\mathbf{1}$ & $\mathbf{2}$ & $\mathbf{3}$ & $\mathbf{4}$ & $\mathbf{5}$ & $\mathbf{6}$ & $\mathbf{7}$ & $\mathbf{8}$ & $\mathbf{9}$ & $\mathbf{1 0}$ \\
\hline TP & 2 & 3 & 2 & 3 & 2 & 2 & 3 & 3 & 2 & 3 \\
\hline FP & 1 & 0 & 1 & 0 & 1 & 1 & 0 & 0 & 1 & 0 \\
\hline FN & 0 & 0 & 2 & 1 & 0 & 1 & 1 & 0 & 0 & 0 \\
\hline
\end{tabular}

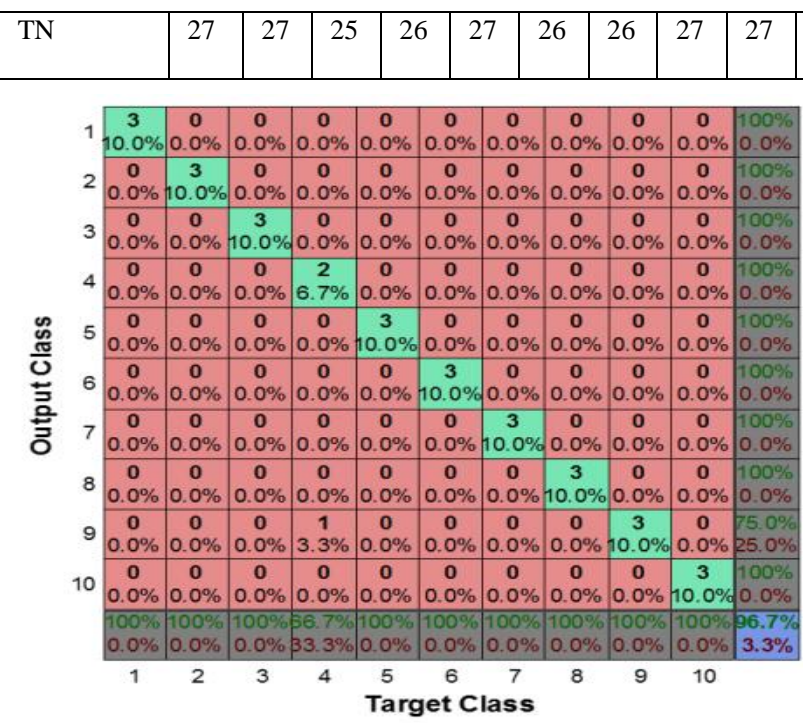

Fig. 5.Confusion matrix plot for the proposed hybrid approach (Face, Iris, and Fingerprint)

Table- IV: Result for proposed hybrid approach (Face, Iris, and Fingerprint)

\begin{tabular}{|l|l|l|l|l|l|l|l|l|l|l|}
\hline \multirow{2}{*}{ Parameter } & \multicolumn{10}{|c|}{ Class } \\
\cline { 2 - 12 } & $\mathbf{1}$ & $\mathbf{2}$ & $\mathbf{3}$ & $\mathbf{4}$ & $\mathbf{5}$ & $\mathbf{6}$ & $\mathbf{7}$ & $\mathbf{8}$ & $\mathbf{9}$ & $\mathbf{1 0}$ \\
\hline TP & 3 & 3 & 3 & 2 & 3 & 3 & 3 & 3 & 3 & 3 \\
\hline FP & 0 & 0 & 0 & 1 & 0 & 0 & 0 & 0 & 0 & 0 \\
\hline FN & 0 & 0 & 0 & 0 & 0 & 0 & 0 & 0 & 1 & 0 \\
\hline TN & 27 & 27 & 25 & 26 & 27 & 26 & 26 & 27 & 27 & 27 \\
\hline
\end{tabular}

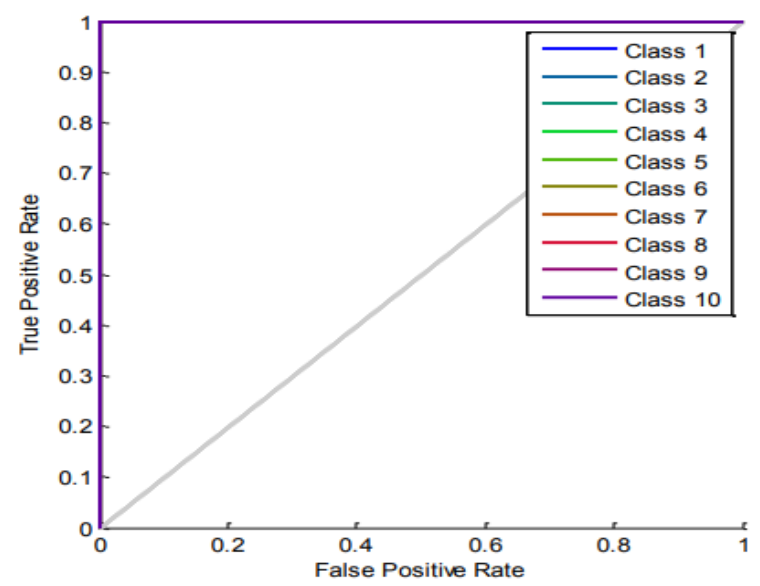

Fig. 6.ROC curve for different classes

Table- V: Various parameters for recognition method

\begin{tabular}{|c|c|c|c|}
\hline $\begin{array}{c}\text { Recognition } \\
\text { method }\end{array}$ & Accuracy & Precision & Sensitivity \\
\hline Face & $98 \%$ & $90 \%$ & $90 \%$ \\
\hline Iris & $96.67 \%$ & $83.3 \%$ & $83.3 \%$ \\
\hline Hybrid(proposed) & $99.33 \%$ & $96.67 \%$ & $96.67 \%$ \\
\hline
\end{tabular}

Published By:

Blue Eyes Intelligence Engineering \& Sciences Publication (c) Copyright: All rights reserved. 


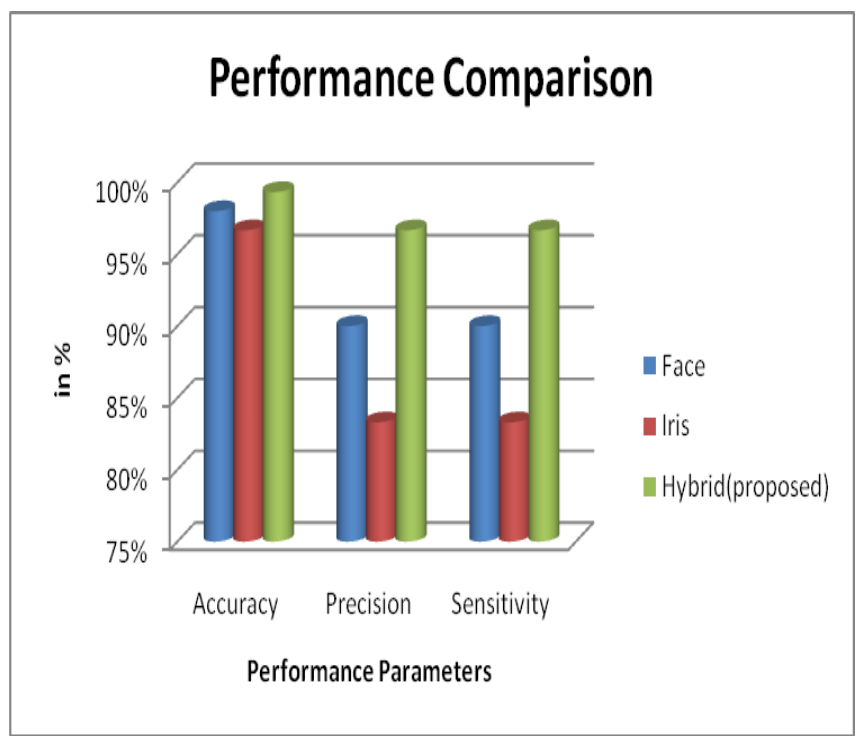

Fig. 7.Comparative Graph for Measuring Performance

\section{CONCLUSION}

Biometrics is an expanding field with a growing body of research that aims to achieve an effective, reliable and timely way of identifying people. The proposed biometric modalities are the face, iris, and fingerprint. This work presented the process of identifying individuals by multimodal biometrics. The main goal is to implement MBS for the identification of individuals where information from three biometric scores (Face, iris and fingerprint) are combined. To get this goal, the proposed work was carried out in several stages. We implemented and tested the face, iris, and fingerprint identification system independently to evaluate their performance and have a clear vision of the robustness of the fusion phase. The multimodal recognition process improves the performance of single-mode systems. Indeed, the tests that we carried out showed the interest of the fusion at the level of the scores. The integration of the data at the level of the correspondence scores by the weighted sum method gives the best result and makes it possible to significantly enhance the performance of the multimodal system. Confusion matrixes for all three recognition have plotted for class 1 to 10 . The accuracy of each class has different values. The overall accuracy for face recognition, iris recognition, and proposed hybrid approach is 98\%, 96.67\% \& 99.33\%, respectively. The overall precision for iris recognition, face recognition \& proposed hybrid approach are $90 \%, 83.3 \%$ \& $96.67 \%$, respectively. The overall sensitivity for iris recognition, face recognition \& proposed hybrid approach has similar values as precision.

The proposed multimodal biometric identification approach may utilize additional biometric approaches like palm-print/finger knuckle to achieve better time complexity.

\section{REFERENCES}

1 A.K. Jain, L. Hong, S. Pankanti, R. Bolle, “An identity authentication system using fingerprints". Proceedings of the IEEE, Vol. 85, issue 9, pp. 1365-1388, 1997.

2 Wang, Yunhong, Tieniu Tan, and Anil K. Jain. "Combining face and iris biometrics for identity verification." In International Conference on Audio-and Video-Based Biometric Person Authentication, pp. 805-813. Springer, Berlin, Heidelberg, 2003.
3 C.Y. Yam, M.S. Nixon, J.N. Carter, "On the Relationship of Human Walking and Running: Automatic Person Identification by Gait" ICPR, pp. 1051-4651, 2002.

4 P. Yan, K. Bowyer, "Empirical Evaluation of Advanced Ear Biometrics", IEEE Computer Society Conference on Computer Vision and Pattern Recognition, pp. 41, 2005.

5 Bhattacharyya, Debnath, Rahul Ranjan, Farkhod Alisherov, and Minkyu Choi. "Biometric authentication: A review." International Journal of u-and eService, Science and Technology 2, no. 3 (2009): 13-28.

6 Sim, Hiew Moi, Hishammuddin Asmuni, Rohayanti Hassan, and Razib M. Othman. "Multimodal biometrics: weighted score level fusion based on nonideal iris and face images." Expert Systems with Applications 41, no. 11 (2014): 5390-5404.

7 Danny Thakkar, "Biometric System Architecture: Getting down the Core", Biometric System, blog.

8 B. Dorizzi, S. Garcia-Salicetti, and L. Allano. "Multimodality In Biosecure: Evaluation On Real Vs. Virtual Subjects”. In: Proceedings of the IEEE International Conference on Acoustics, Speech and Signal Processing (ICASSP), pp. V-V, 2006.

9 Erzin, Engin, Yücel Yemez, and A. Murat Tekalp. "Multimodal speaker identification using an adaptive classifier cascade based on modality reliability." IEEE Transactions on Multimedia 7, no. 5 (2005): 840-852.

10 Delac, Kresimir, and Mislav Grgic. "A survey of biometric recognition methods." In Proceedings. Elmar-2004. 46th International Symposium on Electronics in Marine, pp. 184-193. IEEE, 2004.

11 Komogortsev, Oleg V., Alexey Karpov, Corey D. Holland, and Hugo P. Proença. "Multimodal ocular biometrics approach: A feasibility study." In 2012 IEEE Fifth International Conference on Biometrics: Theory, Applications, and Systems (BTAS), pp. 209-216. IEEE, 2012.

12 Daugman, John G. "Biometric personal identification system based on iris analysis." U.S. Patent 5,291,560, issued March 1, 1994.

13 Chen, Yangyu, and Weigang Zhang. "Iris Liveness Detection: A Survey." In 2018 IEEE Fourth International Conference on Multimedia Big Data (BigMM), pp. 1-7. IEEE, 2018.

14 Shaik, Mehboob. "A novel method identification of automatic iris recognition in an enhanced security system." (2016).

15 Thepade, Sudeep D., and Pooja Bidwai. "Iris recognition using fractional coefficients of transforms, Wavelet Transforms, and Hybrid Wavelet Transforms." In 2013 International Conference on Control, Computing, Communication, and Materials (ICCCCM), pp. 1-5. IEEE, 2013.

16 Galbally, Javier, Jaime Ortiz-Lopez, Julian Fierrez, and Javier Ortega-Garcia. "Iris liveness detection based on quality related features." In 2012 5th IAPR International Conference on Biometrics (ICB), pp. 271-276. IEEE, 2012.

17 Arivazhagan, S., L. Ganesan, and T. Srividya. "Iris recognition using multiresolution transforms." International Journal of Biometrics 1, no. 3 (2009): 254-267.

18 Jain, Anil K., Salil Prabhakar, Lin Hong, and Sharath Pankanti. "FingerCode: a filterbank for fingerprint representation and matching." In Proceedings. 1999 IEEE Computer Society Conference on Computer Vision and Pattern Recognition (Cat. No PR00149), vol. 2, pp. 187-193. IEEE, 1999.

19 Leung, M-T., W. E. Engeler, and P. Frank. "Fingerprint image processing using neural networks." In IEEE TENCON'90: 1990 IEEE Region 10 Conference on Computer and Communication Systems. Conference Proceedings, pp. 582-586. IEEE, 1990.

20 Maio, Dario, and Davide Maltoni. "Direct gray-scale minutiae detection in fingerprints." IEEE transactions on pattern analysis and machine intelligence 19, no. 1 (1997): 27-40.

21 Walia, Gurjit Singh, Tarandeep Singh, Kuldeep Singh, and Neelam Verma. "Robust multimodal biometric system based on optimal score level fusion model." Expert Systems with Applications 116 (2019): 364-376.

22 Shih, F. "Image processing and mathematical morphology". Boca Raton: CRC Press, 2009.

23 Daugman, John G. "Complete discrete 2-D Gabor transforms by neural networks for image analysis and compression." IEEE Transactions on acoustics, speech, and signal processing 36, no. 7 (1988): 1169-1179.

24 Zhou, Zhiping, Huijun Wu, and Qianxing Lv. "A new iris recognition method based on Gabor wavelet neural network." In Intelligent Information Hiding and Multimedia Signal Processing, 2008. IIHMSP'08 International Conference on, pp. 1101-1104. IEEE, 2008.

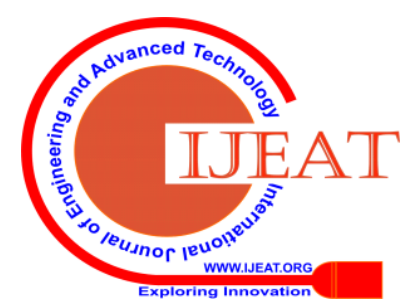




\section{AUTHORS PROFILE}

Shinde Prashant Pandurang pursuing a Master degree in Department of Technology (M.Tech) from Savitribai Phule University, Pune and. His research interests are in the field of Cyber Security and Application Security. He has 6+ years of IT industry experience includes Software development and Application Security. Currently he is working as Assistant Manager in Deloitte for Cyber Risk advisory functions.

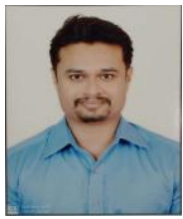

Sable Amol received Bachelor of Engineering (B.E). His research interests are in the field of Information Security Domain and also experienced in Project Management and delivery. He has 15 years of extensive experience in the Information Security domain. He has headed teams that provide information security services to multinational organizations.

Blue Eyes Intelligence Engineering 